\title{
CONOCER PARA AMAR LO QUE TENEMOS. IBERÁ PARA NIVEL INICIAL EN ESCUELAS PERIFERICAS DE LA CIUDAD DE CORRIENTES, ARGENTINA
}

CÉSPEDEZ, Jorge A. ${ }^{1}$; GÓMEZ, Patricia ${ }^{3}$ A.; RUIZ GARCÍA, José A. ${ }^{2}$; CURI, Lucila M. ${ }^{2}$; VILLALVA, Diana ${ }^{3}$; MIÑO, Martha ${ }^{3}$

Palabras Clave: Sistema Iberá, Fauna Autóctona, Nivel Inicial.

Resumen: El presente artículo pretende resolver algunas de las problemáticas que existen en nuestra Comunidad Educativa respecto al conocimiento y estado de Conservación de la Fauna Regional. Se presenta una secuencia de actividades didáctica utilizadas como recursos motivadores para que los niños y niñas del Nivel Inicial fortalezcan y profundicen sus conocimientos relacionados con nuestra fauna autóctona del Sistema Iberá.

Introducción:Este trabajo es el resultado del proyecto de Voluntariado Universitario que proponía la divulgación del conocimiento científico referidos a la composición faunística del Macrosistema Iberá, enfatizando en Anfibios, reptiles, aves y mamíferos, vertebrados considerados en las po- líticas de conservación (sobre todo las aves y mamíferos), y los anfibios y reptiles sobre los que pesa mala fama y supersticiones. A través de este proyecto se propuso contribuir a la educación para la naturaleza en un tema que cala muy hondo en la identidad correntina como es el Iberá, promoviendo en los más pequeños amor por la naturaleza y por la conservación de los recursos naturales y de las especies amenazadas.

El Ibera parece ser inagotable, no sólo por sus dimensiones sino también por su riqueza. Sin embargo, los alumnos correntinos están expuestos a la pérdida de identidad por desconocimiento de las riquezas naturales e históricas que posee nuestra tierra, por lo que sufren el desamor por la misma y los transforma en adultos desinteresados por nuestro patrimonio natural.

Los niños reconocen mediante los medios de comunicación (por ejemplo, televisión) una infinidad de animales exóticos:

\footnotetext{
1- Licenciados en Zoología. Laboratorio de Herpetología. Departamento de Biología. Facultad de Ciencias Exactas y Naturales y Agrimensura. Universidad Nacional del Nordeste. CP. 3400. Corrientes, Argentina. Cel: 3794-790638; e-mail: cespedez@hotmail.com

2- Licenciados en Ciencias Biológicas. Laboratorio de Herpetología. Departamento de Biología. Facultad de Ciencias Exactas y Naturales y Agrimensura. Universidad Nacional del Nordeste.

3- Licenciadas en Educación Inicial. Carrera de Docencia Universitaria. Facultad de Humanidades de la UNNE.
} 


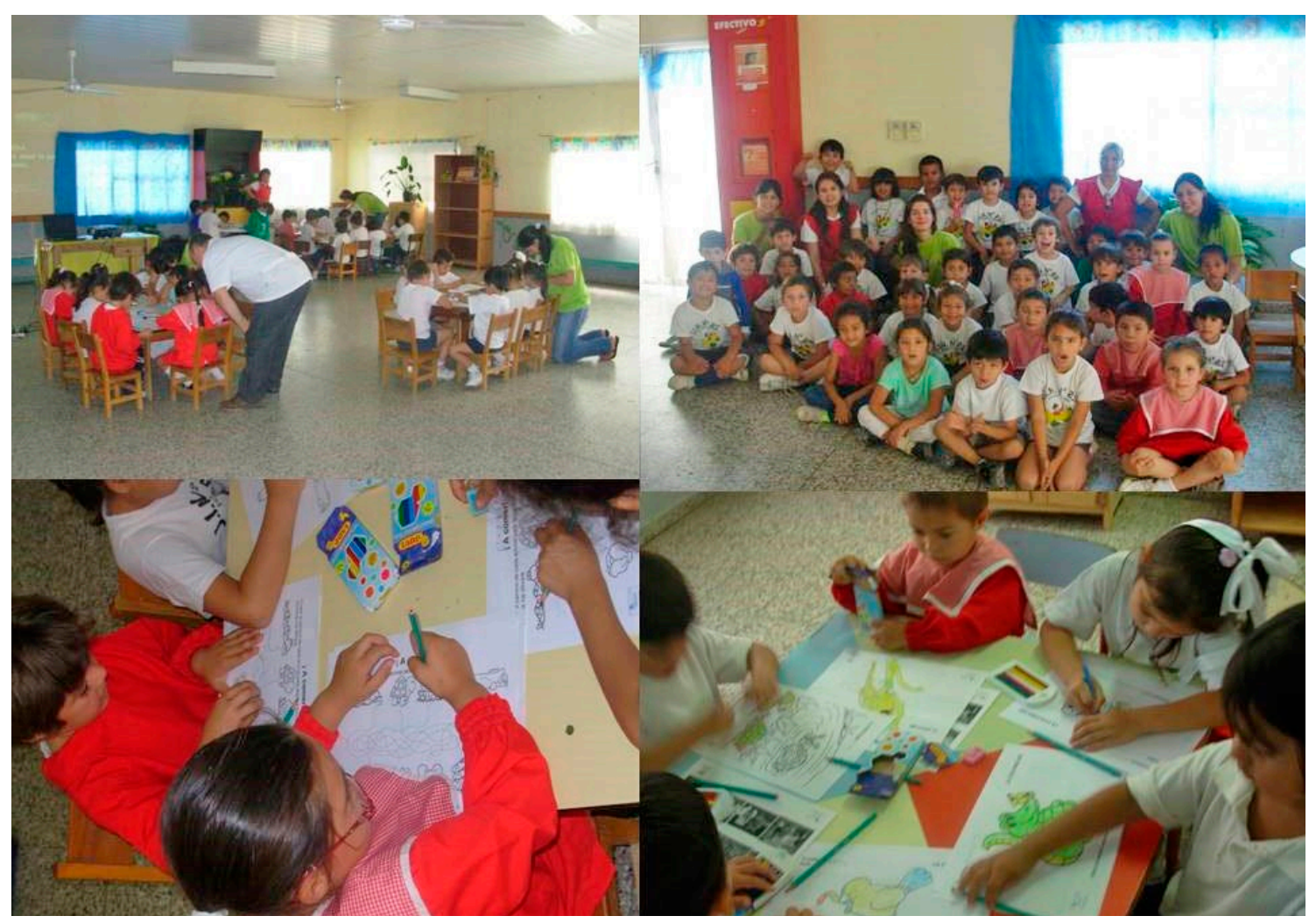

Figura 1

jirafas, elefantes, rinocerontes, topos, entre otros. Sin embargo, desconocen los integrantes de nuestra fauna autóctona (y las consideradas especies banderas) como lo son las vizcachas, carpinchos, ciervos, guazunchos, tatú, y más aún, desconocen las especies de anfibios, reptiles, aves y mamíferos que están vulnerables o consideradas en peligro de extinción (Cuaderno de Herpetología 2012, Suplemento Especial; Aves Argentina/AOP y Secretaría de Desarrollo Sustentable de la Nación 2008, y www.iucnredlist.org/initiatives/mammals).

Partiendo del pensamiento de que no se puede amar lo que no se conoce y por lo tanto no se puede valorar lo que se tiene, se decidió fortalecer y profundizar el conoci- miento de nuestra fauna, informando y educando a quienes se consideran los mejores agentes multiplicadores de conocimientos: "los niños".

La estrategia de enseñanza utilizada en esta experiencia educativa se fundamenta en que los niños y niñas que asisten al Jardín de Infantes o Nivel Inicial, tienen una dinámica que está probada, lo que aprenden en la escuela lo transmiten a sus hogares y en cierta manera presionan a los padres y familiares a indagar y conocer un poco más sobre el tema. Esta experiencia didáctica estuvo dirigida a alumnos de Nivel Inicial de escuelas periféricas o áreas suburbanas del departamento capital de la provincia de Corrientes. Los objetivos de trabajo establecidos fueron: 


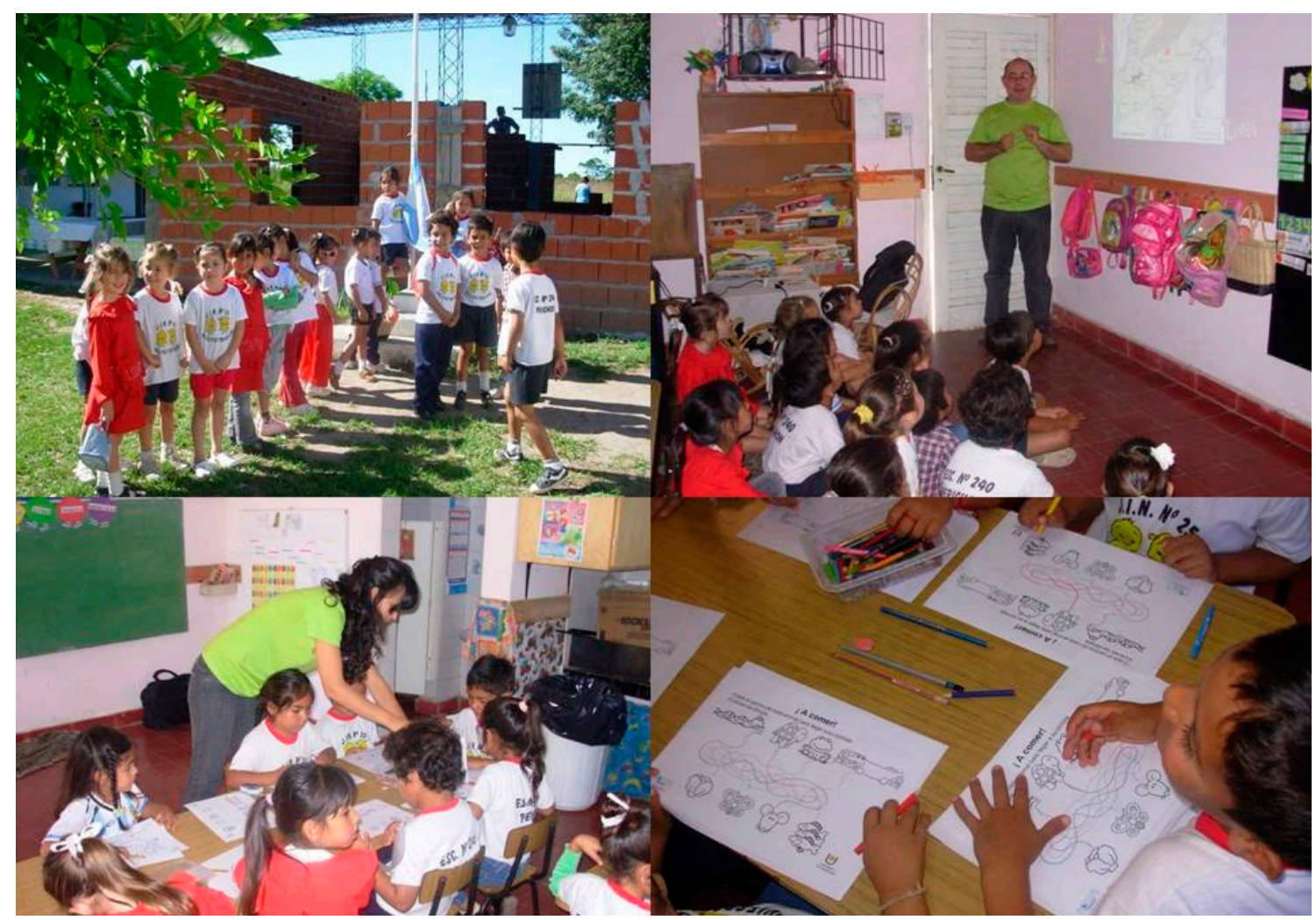

Figura 2

- Fortalecer el grado de conocimiento que poseen los alumnos de Nivel Inicial de Corrientes sobre la fauna regional.

- Promover la valoración de las especies autóctonas (y rescatar los cuentos y leyendas sobre los mismos).

- Informar y formar a los alumnos sobre la importancia del estado de conservación de animales autóctonos.

Metodología y Selección de Actividades: El grupo de trabajo estuvo integrado por docentes, investigadores y alumnos de la carrera de Licenciatura en Ciencias Biológicas de la FaCENA y de la carrera de Especialización en Docencia Universitaria de la UNNE.
El trabajo se desarrolló en el ámbito de la educación formal con trasferencia directa en las siguientes Comunidades Educativas de las zonas periurbanas del Departamento Capital:

- JIN No 25 de las localidades de: Sede Central (Fig. 1), Perichón (Fig. 2) Santa Ana y Paraje Yecohá (Fig. 3).

- JIN No 15 de las escuelas No 640 y No 850 de la Ciudad de Corrientes (Fig. 4).

Las actividades fueron elaboradas $y$ planificadas luego de haber contactado con los docentes de cada establecimiento con la finalidad de conocer las estrategias de enseñanzas utilizadas para abordar temas de la fauna regional.

En visitas posteriores a los establecimientos educativos se les informó a los 


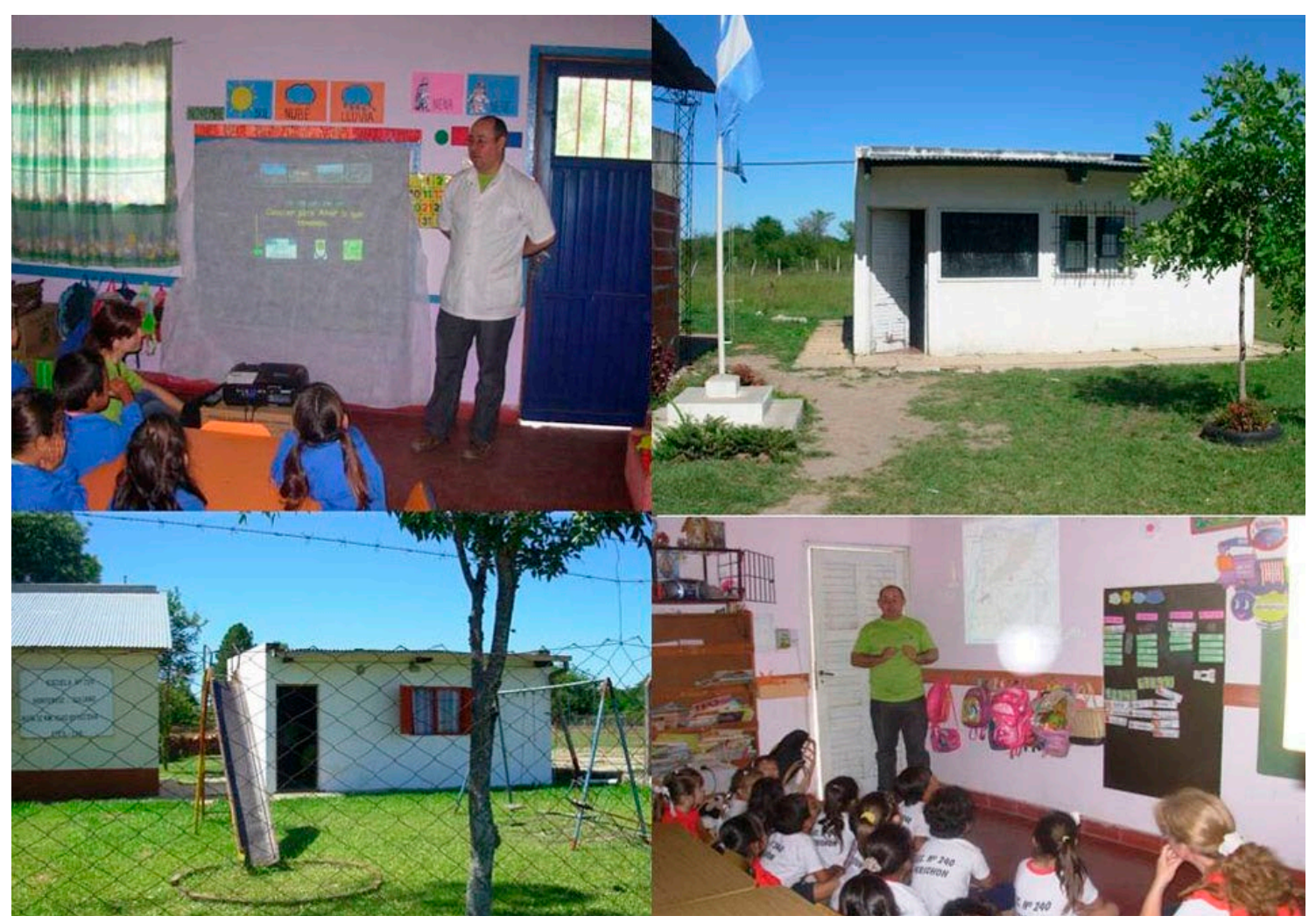

Figura 3

docentes sobre las actividades planificadas a llevar a cabo con los niños y niñas del $\mathrm{Ni}$ vel Inicial. Se les acercó el material didáctico a ser utilizado, y se les explicó acerca de los objetivos que se pretendían alcanzar con la ejecución de este proyecto. También se profundizó sobre la información que los docentes brindan a los niños y niñas sobre la fauna local y la importancia de la misma en su ambiente natural.

Esta experiencia educativa se llevó a cabo en varias jornadas de trabajo. Hubo una primera instancia que consistió en la capacitación de los estudiantes voluntarios, con la finalidad de impartirles charlas informativas y formativas sobre la Biodiversidad de anfibios, reptiles, aves y mamíferos del Sistema Ibera.

En otra jornada, se preparó todo el material didáctico a ser utilizado en los diferentes Jardines de Infantes.

En jornadas posteriores se llevó a cabo el trabajo áulico con los niños y niñas a partir de una introducción motivadora del tema en general, utilizando como herramienta de soporte los recursos visuales que apoyaron la exposición en la cual se realizaron preguntas disparadoras referidas a biodiversidad, biología y estado de conservación de las especies que habitan el Sistema Ibera, acorde al nivel de comprensión de los alumnos, por ejemplo, ¿saben que es el tatú?, ¿cómo son los carpinchos?, ¿saben que comen?, ¿adonde viven?, entre otras. 


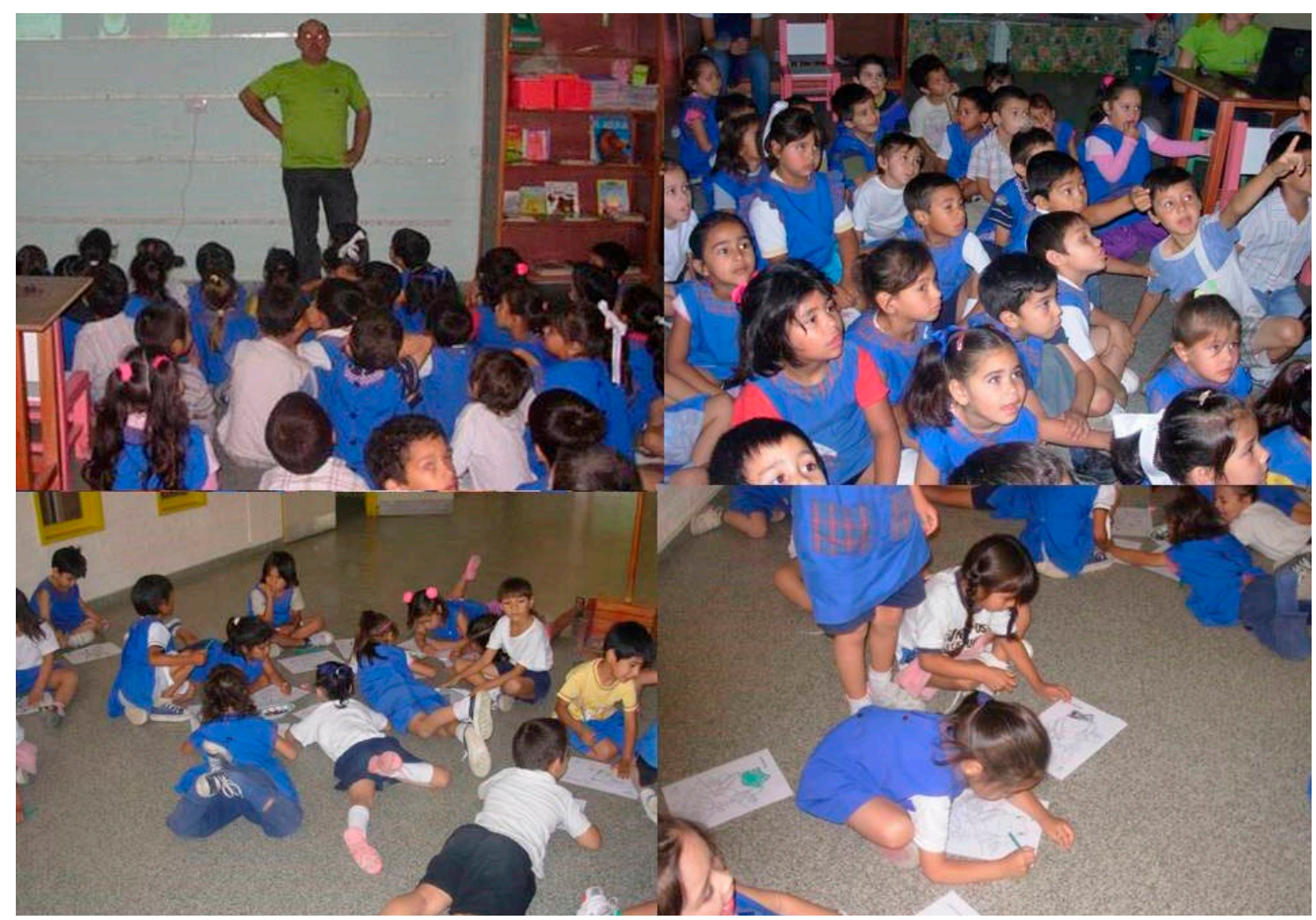

Figura 4

Se implementó el uso de juegos como rompecabezas con figuras de animales de la fauna en general para que los niños y niñas de acuerdo a sus conocimientos básicos seleccionen aquellos pertenecientes a la fauna regional. Incluyéndose también, los mitos y creencias sobre animales de la fauna silvestre que fueron temas de discusión.

Posteriormente, los escolares formaron pequeños grupos dirigidos por los estudiantes voluntarios y se les repartió a cada niño y niña fichas con animales autóctonos y un set de lápices de colores para colorear.

En otro momento de la jornada, se les presentó una caja con tarjetones que tenían impreso numerosas adivinanzas, los cuales eran extraídos por un integrante de cada grupo y respondidos por el resto de los estudiantes.

Resultados: Como cierre de estas actividades, se les entregó a los directivos de cada establecimiento visitado un $\mathrm{CD}$ con fotos y videos de los niños que participaron en las diferentes actividades didácticas y Posters sobre la fauna de anfibios, reptiles, aves y mamíferos del Sistema Ibera y la provincia de Corrientes para ser utilizado en ciclos lectivos posteriores.

Se considera que las actividades realizadas fueron muy positivas para la comunidad educativa quienes compartieron con el grupo responsable de la ejecución del proyecto la experiencia de adquirir conocimientos 
más profundos sobre la fauna autóctona del Sistema Ibera. Esto generó expectativa tanto en los docentes y alumnos de las instituciones como así también a padres, tutores y vecinos de la zona, lográndose rescatar los saberes previos con los que llegan los alumnos al Jardín.

Esta actividad cubrió el objetivo de llegar a cada uno de los lugares de los alumnos actuando los mismos como agentes multiplicadores de la información. Es decir, se convirtieron en el nexo entre la escuela y la familia. Se logró también, revertir con estas actividades la poca o nula importancia que se le da a la conservación de estos animales y la necesidad de crear reservas reales dentro del Sistema para que las especies que allí habitan no se extingan o desaparezcan

Teniendo en cuenta las actividades desarrolladas para la concreción de los objetivos y metas, se considera que éstos se han cumplido de manera altamente satisfactoria:

- Se ordenó el conocimiento que ya poseían los niños sobre la biología de muchas especies de animales destacando el valor de las especies de nuestra fauna local por sobre las aprendidas en la televisión, todas ellas de otros países o continentes, testificándonos de que no exista más confusiones entre las mismas.

- Se transmitió, a través de charlas y material gráfico de divulgación, los conocimientos científicos generados en la Universidad Nacional de Nordeste a fin de contribuir a la conservación de la biodiversidad de Corrientes, y especialmente de uno de los humedales emblemáticos de Argentina: los Esteros del Ibera. Álvarez, et al. (2002 y 2003); Asociación Herpetológica Argenti- na Edición Especial (2012).

- Se brindó información sobre el reconocimiento y biología de especies de reptiles venenosos como no venenosos, con el objeto de desterrar mitos sobre la fauna silvestre.

- Se promovió y fortalecieron los vínculos entre las organizaciones de la sociedad y los grupos académicos de la UNNE.

- Esta experiencia didáctica permitió a una cantidad considerable de alumnos, docentes y Directivos, 487 en total, participar de actividades referidas a la fauna autóctona y poco conocida de nuestro Sistema Iberiano, quienes serán los futuros multiplicadores de la información.

Conclusiones: La experiencia áulica realizada con los alumnos de Nivel Inicial abrió la posibilidad de que estos niños y niñas exploren más de acerca la diversidad, protección y conservación de los animales autóctonos de la zona del Ibera.

Se estimuló en los niños y niñas el deseo de dialogar sobre el tema desarrollado con sus padres y al mismo tiempo el interés de ellos por visitar el parque zoológico de la ciudad con la finalidad de conocer personalmente a ejemplares de las especies tratadas, ya que muchos de ellos solo conocen a los animales autóctonos a través de láminas, libros de cuentos o televisión.

Finalmente, se logró el compromiso de todos los participantes involucrados en esta experiencia áulica generándose acciones multiplicadoras del conocimiento que los docentes pueden abordar con sus respectivos alumnos en el aula y con el resto de la comunidad educativa. 


\section{BIBLIOGRAFÍA}

ÁLVAREZ, Blanca B. (Directora - Coor- ASOCIACIÓN HERPETOLÓGICA ARdinadora); Roberto H. Aguirre; Jorge A. GENTINA 2012. Categorización del EsCéspedez; Alejandra B. Hernando y María E. Tedesco. Colaborador: Oscar Orfeo. 2002. Atlas de Anfibios y Reptiles de las Provincias de Corrientes, Chaco y Formosa (Argentina) I. (Anuros, Cecílidos, Saurios, Amphisbénidos y Serpientes) Ed. EUDENE. (Univ. Nac. Nordeste).156 p. ÁLVAREZ, Blanca B.; Roberto H. Aguirre; Jorge A. Céspedez; Alejandra B. Hernando; y María E. Tedesco. 2003. Herpetofauna del Iberá. 99-178. En: Álvarez B. B. y EUDENE (Eds.). Fauna del Iberá. 375 p. tado de Conservación de la Herpetofauna de la República Argentina. Cuadernos de Herpetología 26 Supl.1: 117-413 pp. LÓPEZ-Lanús, B., P. Grilli, E. Coconier, A. Di Giacomo y R. Banchs. 2008. Categorización de las aves de la Argentina según su estado de conservación. Informe de Aves Argentinas /AOP y Secretaría de Ambiente y Desarrollo Sustentable. Buenos Aires, Argentina. 1a ed. -Buenos Aires: Aves Argentinas AOP. 64 p. www.iucnredlist.org/initiatives/mammals 